\title{
The Hidden Flavors Solution to Solar Neutrino Problem
}

\author{
Aayush Verma \\ India \\ Email: aayushverma6380@gmail.com
}

\begin{abstract}
The Solar Neutrino Problem has been solved, maybe, but still, it is a confusing problem with an undefined solution. I think and We all could think that the mass of the neutrino, its flavor, and the solar problem is connected. In this paper, I have introduced hidden flavors of neutrino and predicted flavors to the whole lepton family with the help of the Solar Neutrino Problem. Hidden flavors are a theoretical prediction to the current solution of the Solar Neutrino Problem. And a lot of reviews are done for the current mechanism and dynamics of the neutrino system.
\end{abstract}




\section{Introduction}

Neutrino is still that one particle in the group of many whose physical properties are unknown to physics. These are neutral particles that interact very rarely with matter. There are 3 kinds of neutrinos i.e electron, muon, and tau, scientifically called flavors. Now it is certainly assumed that mass of these all flavors are nearly just $\left|\Delta m_{1,2}\right|^{2}=0.000079 \mathrm{eV}^{2}$ [1] or sum of the flavors to $0.23 \mathrm{eV}$ or nearly up to $1 \mathrm{eV}$ maybe, actually the mass of the neutrino is still in the findings, different results are being pushed into the theoretical calculations, we get different results for different inputs. And yet, none of the calculations are intuitive or fit into the modern experience of particle and universe.

Usually when neutrinos interact with another particle, then the chemical equation sets out to be

$$
v_{l}+X=l^{-1}+Y
$$

where $l$ is an electron, muon, or tau neutrino, $\mathrm{X}$ is initial and $\mathrm{Y}$ is a final particle, mentioning here that $l$ in the final state will have negative if the $v_{l}$ is the neutral neutrino, otherwise positive for antineutrinos.

And The Solar Problem, more precisely The Solar Neutrino Problem [2][3], is not a problem, it can be solved in just a minute if we know the best and accurate measurement of neutrino's mass and how these flavors behave. So actually the problem arose when our assumptions of neutrino didn't match the actual count by the experiment. One such solution given to the problem is that the flavors automatically change [4]during a neutrino's flight, and the detector at the Homestake experiment [5]was just observing one flavor at that time, being based on the electron neutrino capture process.

$$
v_{e}+{ }^{37} \mathrm{Cl} \rightarrow{ }^{37} \mathrm{Ar}+e^{-}
$$

Neutrino Oscillation [4] is a given scientific name to the constant oscillation of flavors during a flight. It has been theorized that flavors keep changing results because of oscillations during the journey and make our detection even slower and inefficient than it is. The technology advancement to detect neutrino has not yet been much developed, and these oscillations make it even less possible to detect. In sun they are initially electron neutrinos and perform quantum tunneling, as per Standard Solar Model, they are produced much in the proton-proton fusion, which we will discuss in the later section.

\section{Neutrino Oscillation and Interactions}

Neutrino Oscillation, as you know, is an unusual phenomenon that was not introduced until the late twentieth century to physics. This scientific topic was introduced as a solution to the solar neutrino problem. The solution was that, neutrinos have three flavors, as I mentioned above, these flavors constantly change. Neutrino oscillations depend upon the squared mass difference of the neutrinos. Like if sun emits a stream of neutrino, first they will be electron type after tunneling it may be other of two and by reaching the Earth it can be anything between electron, muon, and tau. It is a superposition of mass eigenstates. We will be discussing all the physics under the shadow of 
Quantum Mechanics, so neutrino is a wave-like thing here rather than particle which is preferable for more astronomical studies. So can we choose flavor while observing them, not of course not, this is a basic principle of Quantum Mechanics which erode. The principle states that eigenstates are present in a mere superposition, and we get one arbitrary eigenstate when observing.

Once produced, however, the neutrinos and antineutrinos with given flavors cannot propagate unperturbed, since they are not states with definite mass. They are rather superpositions of states with a given mass, according to certain coefficients $\mathrm{Uj}$, known as leptonic mixing matrix; in formulae.

$$
\begin{aligned}
& \left|v_{l}>=U_{l j}^{*}\right| v_{j}> \\
& \left|\bar{V}_{l}>=U_{l j}\right| \bar{V}_{j}>
\end{aligned}
$$

Well, we are not going any deep to the formulae.

Now why these neutrino oscillations are necessary? So actually we didn't know about them until our solution to the solar neutrino problem. This tells us that neutrinos are not as simple as we think, it could have more surprises for us shortly. There are a lot of implications of flavors oscillations in determining neutrino interactions, but we will not be going deep here on that, but generally, if a particle travels a distance d can say that,

$$
\begin{gathered}
\tau=n * \sigma * d \\
P(d)=\exp (-\tau)=\exp (-d / \lambda)
\end{gathered}
$$

where $\mathrm{P}(\mathrm{d})$ is the probability of interaction over $\sigma$ which is connected to the number of density targets $\mathrm{n}$ and path $\lambda$.

While talking about reactions we should consider reactions of electronic neutrinos and antineutrinos, where $\mathrm{n}$ is neutrons, $\mathrm{p}$ is proton and IBD is inverse beta decay.

$$
\begin{array}{ll}
\hline \beta^{-} \text {decay } & n \rightarrow p+e^{-}+\bar{v}_{e} \\
\beta^{+} \text {decay } & p \rightarrow n+e^{+}+v_{e} \\
\beta^{-} \text {capture } & p+e^{-} \rightarrow n+v_{e} \quad \text { Table. } 1 \\
\beta^{+} \text {capture } & n+e^{+} \rightarrow p+\bar{v}_{e} \\
\text { IBD } & p+\bar{v}_{e} \rightarrow n+e^{+} \\
\text {IBD on } n & n+v_{e} \rightarrow p+e^{-}
\end{array}
$$

We have to understand what is the importance of beta decay for neutrinos, it is essential for neutrinos to held small masses in order to be produced or produce others during beta decay. So we get one prediction from here that Neutrinos should have small masses, it could be more than one million times smaller than an electron. Now, studying leptonic mixing with interactions is very complex, but let's consider one case for IBD reaction which produces the top most probability for neutrinos more precise anti neutrinos to detect.

$$
p+\bar{v}_{e} \rightarrow n+e^{+}
$$


Eq [5] shows that when anti-electron neutrons interact with protons, they produce neutrinos and positrons. Remember that, neutrinos never expose themselves naked, they interact with some particle and then by-products we assume that there are neutrinos, based on observations and theoretical works.

Interactions of neutrinos involves cross-sections, as we have discussed above, in Gargamelle Bubble Chamber, this was too low, attributed to $\bar{v}_{e} e^{-} \rightarrow \bar{v}_{e} e^{-}$. Well, there has been a history of calculating cross-section of neutrinos by interacting with different elements,

(KamLAND, Borexino) [1], (SNO, Super-Kamiokande) [7], (ICARUS) [8], (OMNIS), (MINOS) [9], (COBRA), and (XENON).

That's a lot of neutrinos basic interaction system and oscillations, let's jump to The Solar Neutrino Problem.

\section{The Solar Neutrino Problem}

This is a well-known physics problem related to cosmos-particle physics. First, let's understand the chain by which neutrino travels from the sun to the outside universe. In the Sun, there are many reactions namely, pp reaction, pep reaction, $\mathrm{CNO}$ and so on. But most important is pp reaction, which I have briefly outlined in the introduction [6].

$$
p+p \rightarrow D+e^{+}+v_{e}
$$

This results in the formation of neutrino, more specific electron neutrino. Now for photon, it takes millions of years to reach the surface of the earth, but thanks to quantum tunnelling, neutrinos perform this in seconds.

Now as we have discussed oscillations, these neutrinos will oscillate during their flight, for say let's assume they are coming to Earth, they as eigenstate will be oscillating till our observation. The solar neutrino flux is about $6 \times 10^{11} \mathrm{~cm}^{-2} \mathrm{~s}^{-1}[10]$ at Earth surface.

There is one more type of reactions which yields neutrinos inside the star, but typically not favoured, which are CNO cycles. The CNO I cycle begins with,

$$
\begin{gathered}
{ }^{12} C+p \rightarrow{ }^{13} N+\gamma \\
{ }^{13} N \rightarrow{ }^{13} C+e^{+}+v_{e} \\
{ }^{13} C+p \rightarrow{ }^{14} N+\gamma \\
{ }^{14} N+p \rightarrow{ }^{15} \mathrm{O}+\gamma \\
{ }^{15} \mathrm{O} \rightarrow{ }^{15} N+e^{+}+v_{e} \\
{ }^{15} \mathrm{~N}+p \rightarrow{ }^{12} \mathrm{C}+{ }^{4} \mathrm{He}
\end{gathered}
$$

As we can see the reaction that produced neutrino, now replacing the alpha emission with gamma emission in the last reaction we get CNO II cycle,

$$
\begin{aligned}
& { }^{15} \mathrm{~N}+p \rightarrow{ }^{16} \mathrm{O}+\gamma \\
& { }^{16} \mathrm{O}+p \rightarrow{ }^{17} \mathrm{Fe}+\gamma
\end{aligned}
$$




$$
\begin{aligned}
& { }^{17} \mathrm{Fe} \rightarrow{ }^{17} \mathrm{O}+e^{+}+v_{e} \\
& { }^{17} \mathrm{O}+p \rightarrow{ }^{14} \mathrm{~N}+{ }^{4} \mathrm{He}
\end{aligned}
$$

but this cycle is not frequent in our sun, there are also CNO III and CNO IV, but they are not for our sun. CNO neutrinos give us a chance to understand the $\mathrm{CNO}$ cycle. But unfortunately, due to background events, we get a real challenge to experiment with it. The lists above tell us that there are large numbers of neutrinos coming from the sun to earth, but by the observation, our prediction was not happy with observation. The theoretical prediction never made into reality. Then the solution was proposed, that neutrino oscillates and never states in a single state.

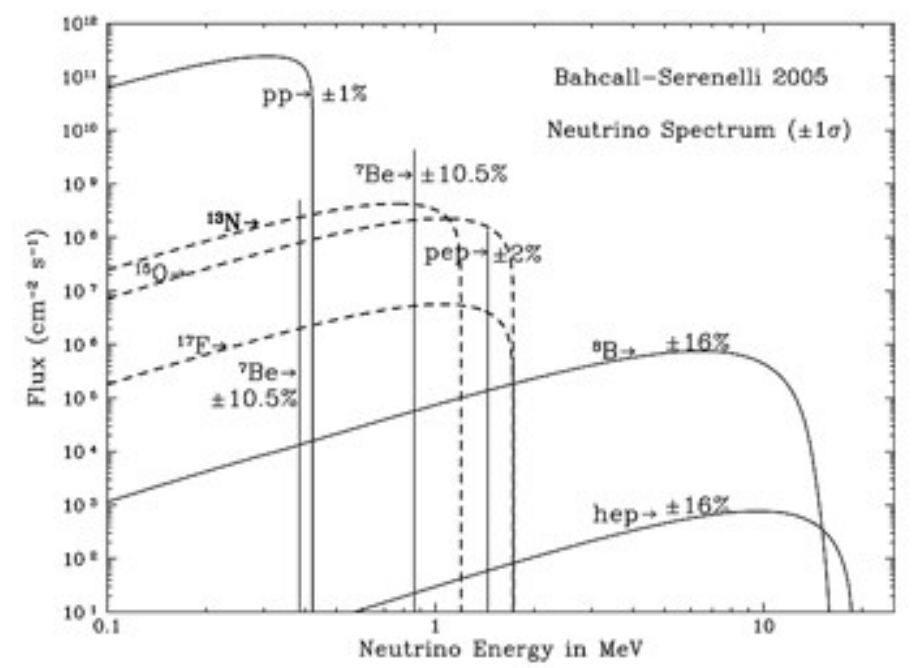

Fig 1. This graph is presentation of various reaction which involves the neutrino creation and operation.

\section{The Arbitrary Solution}

Now the main focus of the paper is this section, where I am about to propose my model of neutrino oscillations. So far, we have maintained our writing in the view of the existing literature, but the current solution to this problem is not yet correct and satisfy me. We know that the sun emits electron neutrinos on a large scale, and various experiments recorded it too less than our theoretical prediction. To create my model, I would, first of all, emphasize my hypothesis on flavors.

\subsection{Is there only 3 flavors?}

It may be again in this paper, but one effective solution to this problem is oscillations, which are merely an eigenstates problem. Neutrinos are emitted and absorbed in weak processes in their flavor eigenstates but travel as mass eigenstates. This is because all mass eigenstate travels with different speed and so that their quantum mechanical wave packets develop relative phase 
shifts that change how they combine to produce a varying superposition of three flavors.

But one thing is still unclear to me, if neutrino oscillates, why in just three flavors? maybe the solution to this query is just, theoretical physics of ours just can imagine three, but there must be more flavors of neutrino, in-fact there could be flavors of also electron and other leptons.

Reason of saying that is if our detector detects electron neutrinos after interacting proton for say,

$$
v_{e}+p \rightarrow e^{-}+n
$$

And in an antielectron neutrino case, it will produce a positron, and then it is easy with our experimental setups to capture those, or more precisely it can be the Homestake experiment. But this is because we know the underlying physics and we prepare our setup according to our physics knowledge, in this vast universe, these high energies can produce many flavors or one flavor can oscillate between hundreds of sub-flavors, not just for neutrinos but can be granted for any other particle. The reason that we don't see them is that because flavors would decay so exponentially or fast to produce another stream of particles. And if the number of neutrino flavors is not just three, then there must be missing leptons in our standard model of particle physics. This is just my hypothesis, but with great intuition, we can imagine hundreds of flavors floating during their flight, but don't interact with any matter or even our detectors.

\subsection{Hidden Flavors}

What I have proposed in section 4.1 is the key explanation of my model. So far my focus is flavors of neutrinos and indeed I would continue so explaining this. My proposed model is that there are not only three flavors of neutrino but many. And the reason that we don't know about those hidden flavors is because they either do not interact a little or their physics is far more complex than our physics understanding. But I will prefer the first one more. Although there can be many probabilities of not finding hidden flavors. At this time, mathematical rigor proof of hidden flavors is a little complex, but I intend to find it. And there can be many solutions as there will be many flavors. As of now, these flavors may be just prediction as our current solution to the solar problem is just the number of theoretical predictions is bigger than the experimental result, so if more than 3 flavors exist, then maybe there would many neutrinos still not observed. Now pointing to the well-known question is very complicated now, but in future, if our maths is reliable, then the mystery of mass and flavors would resolve.

Let me give you a sketch of hidden flavors. These flavors would have very little mass, maybe a little more than negligible so that they don't interact or observed with our devices. These hidden flavors with current 3 flavors constitute the whole neutrino system. This means that our current system of the neutrino is not complete. Maybe some of the hidden flavors can have zero mass but still, constitute the system. So at the end of our discussion, this is my theoretical prediction and can be tested if we have enough technology. At this point saying about the mathematics of this is very hard as I said, but this 
is the most beautiful idea in neutrino if we understand the mechanism but not shortly.

Not only neutrinos but maybe every lepton has flavors, and the lepton we see is just its one flavor. For example, if we observe an electron, we are observing a flavor of the electron. Now the basic question is then why not the characteristics of electron change each time when we observe? This is because we see just one and only one flavor in our finite region. Maybe there is a specified space-time for different flavors. And if these flavors exist, believe me, there is a lot of work to be done in physics!

\section{Conclusion}

First of all, we discussed the neutrino and basis properties of neutrinos. Then we discussed the Neutrino Oscillation as changing the stream of eigenstates. Then we emphasized the whole reaction system of neutrino and talked about the theoretical prediction of numbers of neutrinos flux. Then we get into the solar problem and discussed the solution and in the end, I proposed my model of hidden flavors. Hidden flavors are those flavors whose existence is not yet predicted. And there can be many of these hidden flavors not interacted yet. And then my goal was to give as much information about these hidden flavors in term of the existence physics. 


\section{References}

[1] T. Araki et al. (KamLAND Collaboration) Phys. Rev. Lett. 94, $081801-$ Published 1 March 2005

[2] M. Fukugita and T. Yanagida, Physics of Neutrinos and Applications to Astrophysics, Springer (2003).

[3] Kuo, Tzee-Ke, and James Pantaleone. "Solar-neutrino problem and three-neutrino oscillations." Physical review letters 57.14 (1986): 1805.

[4] Wolfenstein, Lincoln. "Neutrino oscillations in matter." Physical Review D 17.9 (1978): 2369.

[5] Davis, Raymond. "A review of the Homestake solar neutrino experiment." Progress in Particle and Nuclear Physics 32 (1994): 13.

[6] N. Vinyoles et al., A New Generation of Standard Solar Models, APJ 835 202 (2017).

[7] Ahmad, Q. R., et al. "Measurement of day and night neutrino energy spectra at SNO and constraints on neutrino mixing parameters." Physical Review Letters 89.1 (2002): 011302.

[8] Antonello, M., et al. "Measurement of the neutrino velocity with the ICARUS detector at the CNGS beam." Physics Letters B 713.1 (2012): 17-22.

[9] Barger, V., et al. "Neutrino oscillation parameters from MINOS, ICARUS, and OPERA combined." Physical Review D 65.5 (2002): 053016.

[10] Fukuda, Y., et al. "Measurements of the solar neutrino flux from Super-Kamiokande's first 300 days." Physical Review Letters 81.6 (1998): 1158. 